This item was submitted to Loughborough's Research Repository by the author.

Items in Figshare are protected by copyright, with all rights reserved, unless otherwise indicated.

\title{
An adaptive step-size code-constrained minimum output energy receiver for nonstationary CDMA channels
}

PLEASE CITE THE PUBLISHED VERSION

PUBLISHER

(C) IEEE

VERSION

VoR (Version of Record)

\section{LICENCE}

CC BY-NC-ND 4.0

\section{REPOSITORY RECORD}

Yuvapoositanon, Peerapol, and Jonathon Chambers. 2019. "An Adaptive Step-size Code-constrained Minimum Output Energy Receiver for Nonstationary CDMA Channels". figshare.

https://hdl.handle.net/2134/5915. 
This item was submitted to Loughborough's Institutional Repository (https://dspace.lboro.ac.uk/) by the author and is made available under the following Creative Commons Licence conditions.

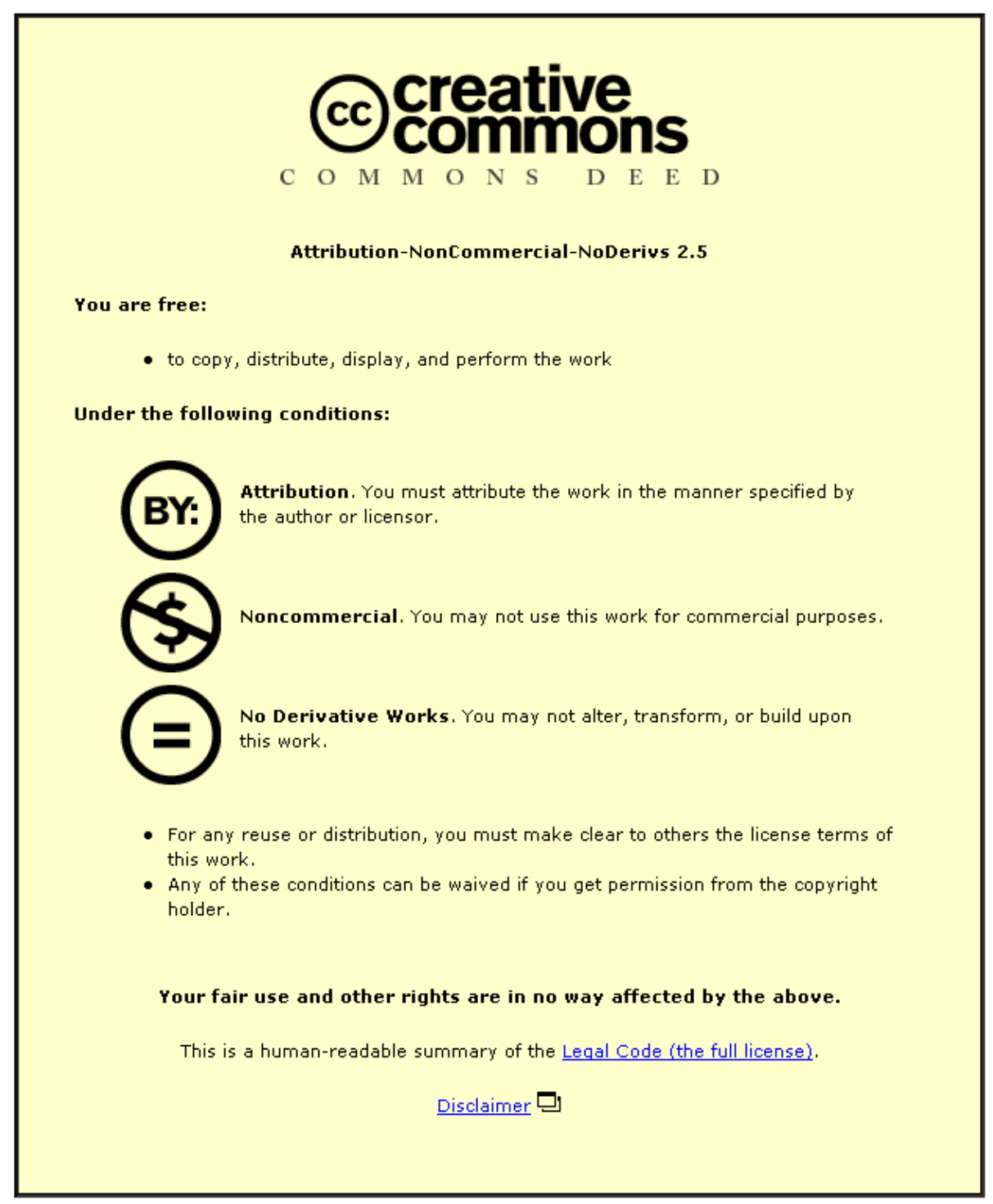

For the full text of this licence, please go to: http://creativecommons.org/licenses/by-nc-nd/2.5/ 


\section{AN ADAPTIVE STEP-SIZE CODE-CONSTRAINED MINIMUM OUTPUT ENERGY RECEIVER FOR NONSTATIONARY CDMA CHANNELS}

\section{Peerapol Yuvapoositanon}

\author{
Department of Electronic Engineering, \\ Mahanakorn University of Technology, \\ Nong-Chok, Bangkok 10530, Thailand \\ Email: peerapol@mut.ac.th
}

\begin{abstract}
The adaptive step-size (AS) code-constrained minimum output energy (CMOE) receiver for nonstationary code-division multiple access (CDMA) channels is proposed. The AS-CMOE algorithm adaptively varies the step-size in order to minimise the CMOE criterion. Admissibility of the proposed method is confirmed via the reformulation of the CMOE criterion as an unconstrained optimisation. The ability of the algorithm to track sudden changes of the channel structure in multipath fading channels is assessed. Sensitivity to the initial values of the step-size and the adaptation rate of the algorithm is also investigated.
\end{abstract}

\section{INTRODUCTION}

In order to ensure stability of the minimum output energy (MOE) algorithms of $[1,2]$ which are based on the stochastic gradient method, the step-sizes must be appropriately chosen. For stationary environments in which channel statistics are assumed static during the adaptation periods, determining an appropriate stepsize for an adaptive algorithm is possible [3]. In practice, however, multiple access interference (MAI) users frequently enter and exit the channel. Therefore, the channel is far from being stationary and computation of a pre-determined step-size is difficult.

In [4], the adaptive step-size strategy for tracking time-varying environments within CDMA channels is successfully applied to the MOE detector presented in [1]. However, the MOE detector tends to cancel the desired signal when the spreading waveform has a mismatch [1]. This is because the MOE criterion is not primarily designed to equalise multipath distortion caused by a dispersive channel. In reality, the multipath distortion, the cause of mismatch, must not be neglected especially when high data rate transmission is required [3].

In this paper, we therefore introduce the adaptive step-size CMOE (AS-CMOE) algorithm for CDMA receivers operating in multipath fading channels. The AS-CMOE algorithm adaptively varies the step-size in order to minimise the CMOE criterion. The AS-CMOE algorithm is derived in the same fashion as the ASMOE algorithm of [4] but with a modification to the constraint in order to operate in multipath channels. The algorithm is blind in the sense that no training data are required. Admissibility of the proposed method is studied by means of the reformulation of the CMOE criterion as an unconstrained optimisation. Simulations confirm the applicability of the algorithms for nonstationary environments in multipath fading CDMA channels. Insensitivity of the algorithms to initial settings of the step-size and the rate of

\section{Jonathon A. Chambers}

\author{
Centre for Digital Signal Processing Research, \\ King's College London, Strand, \\ London WC2R 2LS, United Kingdom \\ Email: jonathon.chambers@kcl.ac.uk
}

step-size adaptation are also shown. The AS-CMOE receiver is shown to perform at least as well as the AS-MOE receivers in both single-path and multipath fading channels.

\section{SIGNAL MODEL}

Consider the real signal model of an additive white Gaussian noise (AWGN) $K$-user synchronous CDMA channel, the baseband received signal is defined as

$$
r(t)=\sum_{i=-\infty}^{\infty} \sum_{k=1}^{K} A_{k} b_{k}(i) c_{k}(t-i T)+w(t),
$$

where $A_{k}$ represents the received amplitude of the $k$ th user. The data bits $b_{k}(i)$ are independent identically distributed (i.i.d.) and $b_{k}(i) \in\{-1,+1\}$. The symbol period is denoted by $T$. The spreading code $c_{k}(t)$ is $N$-dimensional and has unit energy property, i.e., $\left\|c_{k}\right\|^{2}=1$. To include the effect of the channel, the spreading waveform of the $k$ th user $c_{k}(t)$ can be modified to $g_{k}(t)$ $=c_{k}(t) * h_{k}(t)$, where $*$ denotes convolution and $h_{k}(t)$ is the channel response of the $k$ th user which incorporates $A_{k}$ and need not be identical for different users. The AWGN $w(t)$ has power spectral density $\sigma_{w}^{2}$.

The continuous-time received signal $r(t)$ is sampled to form a length- $L_{f}$ received signal vector at the $n$th observation, where $L_{f}$ is the length of a receiver with tap-weight vector $\mathbf{f}$,

$$
\mathbf{r}_{n}=\sum_{k=1}^{K} \mathbf{r}_{n}^{(k)}+\mathbf{w}_{n}=\sum_{k=1}^{K} \mathbf{G}_{k} \mathbf{b}_{k, n}+\mathbf{w}_{n}
$$

where $\mathbf{r}_{n}^{(k)}$ denotes the received signal of the $k$ th user, $\mathbf{G}_{k}$ the combined code-channel response matrix of the $k$ th user, $\mathbf{b}_{k, n}=$ $\left(b_{k, n+L_{b}-1}, \cdots, b_{k, n}\right)^{T}$ with $L_{b}=\left\lceil\frac{L_{f}+L_{h}-1}{N}\right\rceil$ and $\mathbf{w}_{n}=\left(w_{n N+L_{f}-1}, \cdots, w_{n N}\right)^{T}$. Note that

$$
\mathbf{G}_{k}=\mathbf{C}_{k} \mathbf{H}_{k},
$$

where the $L_{f} \times L_{b} L_{h}$ code matrix $\mathbf{C}_{k}$

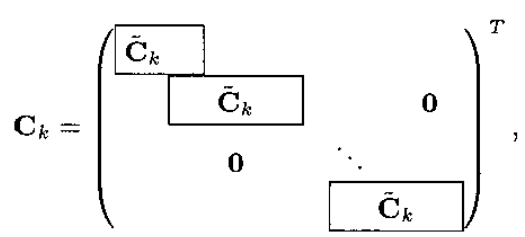


where $\quad \tilde{\mathbf{C}}_{k} \quad$ represents the block of delayed copies of the code sequence of the $k$ th user with dimension $L_{h} \times N+L_{h}-1$

$$
\tilde{\mathbf{C}}_{k}=\left(\begin{array}{ccccc}
0 & \cdots & c_{k, N-1} & \cdots & c_{k, 0} \\
\vdots & \nearrow & & \nearrow & \vdots \\
c_{k, N-1} & \cdots & c_{k, 0} & \cdots & 0
\end{array}\right)
$$

and $\tilde{\mathbf{C}}_{k}$ denotes the code matrix $\tilde{\mathbf{C}}_{k}$ with the first $L_{h}-1$ columns truncated. Define the length $L_{h}$ channel response vector for the $k$ th user, $\mathbf{h}_{k}=\left(h_{0}^{(k)}, \cdots, h_{L_{h}-1}^{(k)}\right)^{T}$, the $L_{b} L_{h} \times L_{b}$ channel matrix $\mathbf{H}_{k}$ and the $L_{f} \times L_{b}$ combined channel matrix $\mathbf{G}_{k}$ are defined as

$\mathbf{H}_{k}=\left(\begin{array}{ccc}\mathbf{h}_{k} & & \mathbf{0} \\ & \ddots & \\ \mathbf{0} & & \mathbf{h}_{k}\end{array}\right), \mathbf{G}_{k}=\left(\begin{array}{ccc}g_{k, N-1} & \cdots & g_{k, L_{b} N-1} \\ \vdots & \ddots & \vdots \\ g_{k, N-L_{f}} & \cdots & g_{k, L_{b} N-1}\end{array}\right)$.

For brevity, we shall consider the first user as the desired user and drop the subscript $k$ in all variables involving the first user.

\section{ADAPTIVE STEP-SIZE CMOE ALGORITHM}

We consider the CMOE criterion for the AS-CMOE algorithm

$$
\min _{\mu} J_{n} \quad \text { s.t. } \quad\left(\mathbf{d}_{n}^{\mu}\right)^{T} \mathbf{C}=(1,0, \cdots, 0) \text {, }
$$

where $J_{n}=E\left\{\left(\left(\mathbf{d}_{n}^{\mu}\right)^{T} \mathbf{r}_{n}\right)^{2}\right\}, \mathrm{C}$ represents the code-constrained matrix described in (4), $\mu$ is the step-size of the algorithm and $\mathbf{d}_{n}^{\mu}$ is the tap weight vector of the fixed step-size CMOE algorithm. The update rule for $\mathbf{d}_{n}^{\mu}$ is given by

$$
\mathbf{d}_{n+1}^{\mu}=\mathbf{d}_{n}^{\mu}-\mu \boldsymbol{\Pi}_{\mathbf{C}}^{\perp} \mathbf{r}_{n} \mathbf{r}_{n}^{T} \mathbf{d}_{n}^{\mu},
$$

where $\boldsymbol{\Pi}_{\mathbf{C}}^{\perp}=\mathbf{I}-\boldsymbol{\Pi}_{\mathbf{C}}$ and $\boldsymbol{\Pi}_{\mathbf{C}} \triangleq \mathbf{C}\left(\mathbf{C}^{T} \mathbf{C}\right)^{-1} \mathbf{C}^{T}$. Differentiating $J_{n}$ in (6) with respect to $\mu$ at time $n$, denoted by $\mu_{n}$, results in $\left.\frac{\partial J_{n}}{\partial \mu}\right|_{\mu=\mu_{n}}=2 E\left\{\mathbf{d}_{n}^{T} \mathbf{r}_{n} \mathbf{r}_{n}^{T} Y_{n}\right\}$, where $Y_{n}=\partial \mathbf{d}_{n} /\left.\partial \mu\right|_{\mu=\mu_{n}}$ and $\mathbf{d}_{n}$ is the estimate of the tap-weight at time $n$. Replacing $\mu$ and $\mathbf{d}_{n}^{\mu}$ in (7) with $\mu_{n}$ and $\mathbf{d}_{n}$ respectively, we arrive at

$$
\mathbf{d}_{n+1}=\mathbf{d}_{n}-\mu_{n} \boldsymbol{\Pi}_{\mathbf{C}}^{\perp} \mathbf{r}_{n} \mathbf{r}_{n}^{T} \mathbf{d}_{n},
$$

where $\mu_{n}$ is updated by

$$
\mu_{n+1}=\left[\mu_{n}-\alpha \mathbf{d}_{n}^{T} \mathbf{r}_{n} \mathbf{r}_{n}^{T} Y_{n}\right]_{\mu-}^{\mu+},
$$

and $[\cdot]_{\mu_{-}}^{\mu_{+}}$denotes the truncation to the limits of the range $\left[\mu_{-}, \mu_{+}\right]$ and $\alpha>0$ denotes the adaptation rate of $\mu_{n}$. The update rule of $Y_{n}$ is given by

$$
\begin{aligned}
Y_{n+1} & =Y_{n}-\mathbf{r}_{n}^{T} \mathbf{d}_{n} \mathbf{r}_{n}-\mu_{n} \mathbf{r}_{n}^{T} Y_{n} \mathbf{r}_{n} \\
& =Y_{n}-\mathbf{r}_{n}^{T} \mathbf{d}_{n} \Pi_{\mathrm{C}}^{\perp} \mathbf{r}_{n}-\mu_{n} \mathbf{r}_{n}^{T} Y_{n} \boldsymbol{\Pi}_{\mathbf{C}}^{\perp} \mathbf{r}_{n} \\
& =\left[\mathbf{I}-\mu_{n} \mathbf{r}_{n} \mathbf{r}_{n}^{T}\right] Y_{n}+\mu_{n} \mathbf{r}_{n}^{T} Y_{n} \Pi_{\mathbf{C}} \mathbf{r}_{n}-\mathbf{r}_{n}^{T} \mathbf{d}_{n} \Pi_{\mathbf{C}}^{\perp} \mathbf{r}_{n},
\end{aligned}
$$

where the second equality results from replacing the received signal $\mathbf{r}_{n}$ with its projection upon the complement of the range space spanned by the columns of the code-constrained matrix $\mathbf{C}, \mathbf{\Pi}{ }_{\mathbf{C}} \mathbf{r}_{n}$. Equations (8), (9) and (10) constitute the adaptive step-size CMOE (AS-CMOE) algorithm. For the fixed- $\mu$ process, $\mu_{n} \triangleq \mu$ and (10) becomes $Y_{n+1}^{\mu}=\left[\mathbf{I}-\mu \mathbf{r}_{n} \mathbf{r}_{n}^{T}\right] Y_{n}^{\mu}+\mu \mathbf{r}_{n}^{T} Y_{n}^{\mu} \boldsymbol{\Pi}_{\mathbf{C}} \mathbf{r}_{n}-\mathbf{r}_{n}^{T} \mathbf{d}_{n}^{\mu} \boldsymbol{\Pi}_{\mathbf{C}}^{\perp} \mathbf{r}_{n}$, Admissibility of using both $Y_{n}^{\mu}$ and $Y_{n}$ in the CMOE criterion (6) is confirmed by the results in Section 4 and 5 .

\section{REFORMULATION OF THE CMOE CRITERION AS AN UNCONSTRAINED OPTIMISATION}

We follow [4] in finding an unconstrained version of the codeconstrained MOE (CMOE) cost. The CMOE criterion of [2] for the fixed- $\mu$ process is given by

$$
\min _{\mathbf{d}_{n}^{\mu}} J_{n} \quad \text { s.t. } \quad\left(\mathbf{d}_{n}^{\mu}\right)^{T} \mathbf{C}=(1,0, \cdots, 0) .
$$

By solving for any one of the element of $\mathbf{d}_{n}^{\mu}$, the constrained optimisation problem of (11) can be transformed into an unconstrained one [4]. Resorting to the constraint in (11), the first element of $\mathbf{d}_{n}^{\mu}$ is given by

$$
d_{n, 1}^{\mu}=\frac{1}{c_{1,1}}\left[1-\sum_{i=2}^{L_{f}} c_{i, 1} d_{n, i}^{\mu}-\sum_{j=2}^{L_{b} L_{h}}\left(\mathbf{d}_{n}^{\mu}\right)^{T} \mathcal{C}^{(j)}\right]
$$

where $c_{i, j}$ denotes the $i, j$ th element of $\mathbf{C}$ and $\mathcal{C}^{(j)}$ is the $j$ th column vector of $\mathbf{C}$, i.e.,

$\mathbf{C} \triangleq\left(\mathcal{C}^{(1)} \cdots \mathcal{C}^{\left(L_{b} L_{h}\right)}\right)=\left(\begin{array}{ccc}c_{1,1} & \cdots & c_{1, L_{b} L_{h}} \\ c_{2,1} & \cdots & c_{2, L_{b} L_{h}} \\ \vdots & \ddots & \vdots \\ c_{L_{f}, 1} & \cdots & c_{L_{f}, L_{b} L_{h}}\end{array}\right)(13)$

The tap-weight vector of the receiver at time $n$ is also redefined to $\mathbf{d}_{n}^{\mu}=\left(d_{n, 1}^{\mu}, \cdots, d_{n, L_{f}}^{\mu}\right)^{T}=\left(d_{n, 1}^{\mu},\left(\overline{\mathbf{d}}_{n}^{\mu}\right)^{T}\right)^{T}$ and the received signal vector $\mathbf{r}_{n}=\left(r_{n, 1}, \cdots, r_{n, L_{f}}\right)^{T}=\left(r_{n, 1}, \overline{\mathbf{r}}_{n}^{T}\right)^{T}$ where $L_{f}$ is the length of the tap-weight vector. The argument of (11) is therefore given by

$$
\begin{aligned}
\left(\mathbf{d}_{n}^{\mu}\right)^{T} \mathbf{r}_{n} & =d_{n, 1}^{\mu} r_{n, 1}+\left(\overline{\mathbf{d}}_{n}^{\mu}\right)^{T} \overline{\mathbf{r}}_{n} \\
& =\frac{r_{n, 1}}{c_{1,1}}-\frac{1}{c_{1,1}}\left(\sum_{i=2}^{L_{f}} c_{i, 1} d_{n, i}^{\mu} r_{n, 1}+\right. \\
& \left.\sum_{j=2}^{L_{b} L_{n}}\left(\mathbf{d}_{n}^{\mu}\right)^{T} \mathcal{C}^{(j)} r_{n, 1}\right)+\left(\overline{\mathbf{d}}_{n}^{\mu}\right)^{T} \overline{\mathbf{r}}_{n} \\
& \triangleq \mathcal{Y}_{n}-\mathcal{F}_{n}^{T} \mathcal{R}_{n},
\end{aligned}
$$

where $\overline{\mathcal{C}}^{(j)}=\left(c_{2, j}, \cdots, c_{L_{f}, j}\right)^{T}, \mathcal{Y}_{n} \triangleq-\frac{\left[1-\sum_{j=2}^{L_{b} L_{h}} c_{1, j} d_{n, 1}^{\mu}\right] r_{n, 1}}{c_{1,1}}$, $\mathcal{R}_{n} \triangleq \overline{\mathbf{r}}_{n}-\frac{1}{c_{1,1}} \sum_{j=1}^{L_{b} L_{h}} \overline{\mathcal{C}}^{(j)} r_{n, 1}$, and $\mathcal{F}_{n} \triangleq \overline{\mathbf{d}}_{n}^{\mu}$. An unconstrained optimisation version of (11) is therefore given by

$$
\min _{\overline{\mathbf{d}}_{n}^{\mu}} J_{n} \quad \text { where } \quad J_{n}=E\left\{\left(\mathcal{Y}_{n}-\mathcal{F}_{n}^{T} \mathcal{R}_{n}\right)^{2}\right\} .
$$

Consider a fixed step-size system,

$$
\mathcal{F}_{n+1}^{\mu}=\mathcal{F}_{n}^{\mu}+\mu \boldsymbol{R}_{n} e_{n}(\mu)
$$

where $e_{n}(\mu) \triangleq \mathcal{Y}_{n}-\mathcal{F}_{n}^{\mu T} \mathcal{R}_{n}$. The unconstrained optimisation for the fixed step-size system (16) is given by

$$
\min _{\mu} E\left\{\left(\mathcal{Y}_{n}-\mathcal{F}_{n}^{\mu T} \mathcal{R}_{n}\right)^{2}\right\}=\min _{\mu} E\left\{e_{n}^{2}(\mu)\right\}
$$

An unconstrained version of the adaptive step-size CMOE (ASCMOE) algorithm is given by

$$
\begin{aligned}
\mathcal{F}_{n+1} & =\mathcal{F}_{n}+\mu_{n} \mathcal{R}_{n} e_{n} \\
\mu_{n+1} & =\left[\mu_{n}+\alpha e_{n} \boldsymbol{\mathcal { R }}_{n}^{T} Y_{n}\right]_{\mu_{-}}^{\mu_{+}} \\
Y_{n+1} & =\left[\mathbf{I}-\mu \mathcal{R}_{n} \boldsymbol{\mathcal { R }}_{n}^{T}\right] Y_{n}+\mathcal{R}_{n} e_{n}
\end{aligned}
$$


where $e_{n}=\mathcal{Y}_{n}-\mathcal{F}_{n}^{T} \mathcal{R}_{n}$. Note that since the AS-MOE criterion of [4] uses the constraint proposed by Honig et al. [1], the constraint in (11) is therefore modified to $\left(\mathbf{d}_{n}^{\mu}\right)^{T} \mathcal{C}^{(1)}=1$. Consequently, the parameters in (14) are modified as follows for the AS-MOE criterion

$$
\mathcal{Y}_{n} \triangleq-\frac{r_{n, 1}}{c_{1,1}}, \quad \mathcal{R}_{n} \triangleq \overline{\mathbf{r}}_{n}-\frac{\overline{\mathcal{C}}^{(1)} r_{n, 1}}{c_{1,1}}, \mathcal{F}_{n} \triangleq \overline{\mathbf{d}}_{n}^{\mu},
$$

which are identical to those shown in Section IV A. of [4]

\section{ADMISSIBILITY OF $Y_{N}^{\mu}$ AND $Y_{N}$ PROCESSES}

For a stationary system with small enough $\mu$, the meaning of $Y_{n}^{\mu}$ is interpreted by the approximation of estimation of finite difference errors as [5]. For $\Delta>0, \mu+\Delta \leq \mu_{+}$, define $\delta \mathcal{F}_{n}^{\mu, \Delta}=$ $\frac{\mathcal{F}_{n}^{\mu+\Delta}-\mathcal{F}_{n}^{\mu}}{\Delta}$. Therefore,

$$
\begin{aligned}
\delta \mathcal{F}_{n+1}^{\mu, \Delta}=\delta \mathcal{F}_{n}^{\mu, \Delta}-\mu \mathcal{R}_{n} \boldsymbol{R}_{n}^{T} \delta \mathcal{F}^{\mu, \Delta} \\
+\mathcal{R}_{n}\left(\mathcal{Y}_{n}-\mathcal{R}_{n}^{T} \mathcal{F}_{n}^{\mu}\right)-\Delta \mathcal{R}_{n} \mathcal{R}_{n}^{T} \delta \mathcal{F}_{n}^{\mu, \Delta}
\end{aligned}
$$

Similarly to the study of $Y_{n}^{\mu}$ for non-blind AS algorithms as shown in Appendix $A$ of [5], it is shown that $\lim \sup _{n} E\left\{\left(\delta \mathcal{F}_{n}^{\mu, \Delta}\right)^{2}\right\} \leq$ $O\left(1 / \mu^{2}\right)$ converges uniformly in $\Delta$ for small enough $\mu$. Define $\mathcal{D}_{n}^{\mu, \Delta}=\delta \mathcal{F}_{n}^{\mu, \Delta}-\tilde{Y}_{n}^{\mu}$ where $\tilde{Y}_{n}^{\mu}=\partial \mathcal{F}_{n}^{\mu} /\left.\partial \mu\right|_{\mu=\mu_{n}}$, therefore

$$
\mathcal{D}_{n+1}^{\mu, \Delta}=\mathcal{D}_{n}^{\mu, \Delta}-\mu \mathcal{R}_{n} \mathcal{R}_{n}^{T} \mathcal{D}_{n}^{\mu, \Delta}-\Delta \mathcal{R}_{n} \mathcal{R}_{n}^{T} \delta \mathcal{F}_{n}^{\mu, \Delta} .
$$

By using the above estimate for $E\left\{\left(\delta \mathcal{F}_{n}^{\mu, \Delta}\right)^{2}\right\}$ and following the same derivation as in Appendix $A$ of [5], it can be shown that $\lim \sup _{n} E\left\{\left(\mathcal{D}_{n}^{\mu, \Delta}\right)^{2}\right\}=O(\Delta / \mu) O\left(1 / \mu^{2}\right)$. This means that for the stationary system and small enough $\mu, \lim _{\Delta \rightarrow 0} E\left\{\left(\bar{Y}_{n}^{\mu}-\right.\right.$ $\left.\left.\frac{\mathcal{F}_{n}^{\mu+\Delta}-\mathcal{F}_{n}^{\mu}}{\Delta}\right)^{2}\right\}=0$. Therefore, $\tilde{Y}_{n}^{\mu}$ can be interpreted as a mean square derivative [5], i.e., as $\Delta \rightarrow 0$,

$$
\frac{E\left\{f\left(\mathcal{F}_{n}^{\mu+\Delta}\right)\right\}-E\left\{f\left(\mathcal{F}_{n}^{\mu}\right)\right\}}{\Delta} \rightarrow E\left\{f_{\mathcal{F}}^{\prime}\left(\mathcal{F}_{n}^{\mu}\right) \tilde{Y}_{n}^{\mu}\right\}
$$

where $f(\cdot)$ is a differentiable function [5] and $f_{\mathcal{F}}^{\prime}(\cdot)$ is the derivative of $f(\cdot)$ with respect to $\mathcal{F}$. Define $f\left(\mathcal{F}_{n}^{\mu}\right)=\left[\mathcal{Y}_{n}-\mathcal{F}_{n}^{T} \mathcal{R}_{n}\right]^{2}$, it is shown that [5]

$$
E\left\{f_{\mathcal{F}}^{\prime}\left(\mathcal{F}_{n}^{\mu}\right) \tilde{Y}_{n}^{\mu}\right\}=\frac{\partial E\left\{f\left(\mathcal{F}_{n}^{\mu}\right)\right\}}{\partial \mu} .
$$

By using the relationship (14) and the results from Section $4, f\left(\mathcal{F}_{n}^{\mu}\right)$ $\triangleq f\left(\mathbf{d}_{n}\right)$. Therefore,

$$
E\left\{f_{\mathrm{d}}^{\prime}\left(\mathbf{d}_{n}^{\mu}\right) Y_{n}^{\mu}\right\}=\frac{\partial E\left\{f\left(\mathbf{d}_{n}^{\mu}\right)\right\}}{\partial \mu} .
$$

Hence, the admissibility of $Y_{n}^{\mu}$, and also $Y_{n}$, as a mean square derivative for the AS-CMOE algorithm is therefore validated.

\section{SIMULATIONS}

We considered a synchronous CDMA system with spreading gain 31. Gold sequences were used to generate the spreading codes for all users. The background noise was zero mean AWGN with $S N R=20 \mathrm{~dB}$ referenced to the desired (first) user. Six equal-power $20 \mathrm{~dB}$ MAI users were in the channel at time zero. At time $n=$
500 , another four $30 \mathrm{~dB} \mathrm{MAI}$ users were added to the channel. At time $n=1000$, two of the $20 \mathrm{~dB}$ MAI users and three of the $30 \mathrm{~dB}$ MAI users exited the channel. Averaged signal-tointerference plus noise (SINR) was used as a performance measure and all SINR plots were averaged over 100 Monte-Carlo runs. The performances of the AS-MOE [4] receiver and the proposed ASCMOE receiver are compared in single-path and multipath fading scenarios. The lengths of both receivers were chosen to be $2 \mathrm{~N}$ and were initialised with the spreading code of the first user followed by $N$ zeroes. Parameters for both algorithms were identically assigned as follows. The initial step-sizes were set at $\mu_{0}=10^{-4}$, the adaptation rates $\alpha$ were $5 \times 10^{-8}$ and $Y_{0}$ were initialised at $0.1(1, \cdots, 1)^{T}$. The upper and lower step-size limits $\mu_{+}, \mu_{-}$ were respectively $10^{-2}$ and 0 .

For the case of the single-path channel, the SINR plots are shown in Fig. 1 a). Both AS algorithms perform almost identical in the case of a non-near-far, single-path stationary channel. As four near-far MAI users enter the channel at time $n=500$, both ASMOE and AS-CMOE are able to track the channel variation with noticeable degradation in SINR level. In the multipath case, fiveray multipath fading channels were assumed for all users where the probability of the location of the delay of the last four rays were uniformly distributed over $\left[0,5 T_{c}\right)$ with standard deviation 0.3. The SINR performances of both receivers in the multipath channel are shown in Fig. 1 b). It is apparent that the performance of the AS-MOE receiver is highly dependent upon the multipath dispersion. The AS-MOE receiver still attains a degraded SINR level even when some users exit the channel at time $n=1000$. For AS-CMOE, the additional constraint prevents signal cancellation. The SINR performance confirms the ability of the AS-CMOE to operate in multipath channels.

Fig. 2 a)-b) show respectively three trajectories of different initialised step-sizes of the AS-CMOE algorithm for single-path and multipath channels. For the single-path channel in Fig. 2 a), the trajectories are driven up to the peak and gradually decrease to a small value. A decrease in the value of the step-size is associated with an increase of the SINR of the AS-CMOE receiver as shown in Fig. 1 b). After $n=1000$, all trajectories stay at the same level. In Fig. 2 b), the trajectories of AS-CMOE for the fiveray multipath channel are shown. At $n=500$, sudden changes in the trajectories for both algorithms are less noticeable as compared to those shown in Fig. 2 a). With the 100 -fold variation in $\mu_{0}=\left\{10^{-5}, 10^{-4}, 10^{-3}\right\}$, all trajectories of the step-sizes for AS-CMOE in Fig. 2 a)-b) show an approximately identical behaviour in convergence even after the occurrence of the sudden changes in the channel. It is noticed that the algorithm converges slightly faster at large initial setting of step-sizes. A similar result has also been observed for the adaptive step-size LMS algorithm shown in [6].

In order to test the effect of $\alpha$ upon the algorithm, we considered the performance of AS-CMOE in a dynamic environment where different user groups switched between three user groups according to a Markov chain. The dynamic environment was set similarly to that of [4] with minor modification. The power of the first user was set such that its SNR is $20 \mathrm{~dB}$ and the MAI user groups were designated as follows. User group 1 consisted of 17 MAI users transmitting at $20 \mathrm{~dB}$ and two MAI users transmitting at $30 \mathrm{~dB}$. User group 2 consisted of $29 \mathrm{MAI}$ users transmitting at $20 \mathrm{~dB}$. Finally, user group 3 consisted of eight MAI users transmitting at $20 \mathrm{~dB}$ and one MAI user transmitting at $30 \mathrm{~dB}$. The MAI structure was constructed according to a Markov chain $x[n]$ with 


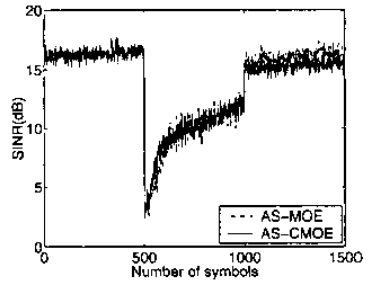

a)

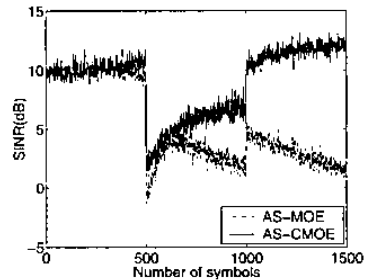

b)
Fig. 1. The averaged SINR in $\mathrm{dB}$ for AS-MOE [4] and AS-CMOE receivers in a) single-path and b) multipath channels. Both the ASMOE and AS-CMOE receivers show comparable performance in tracking variations of the single-path channel. For the multipath channel, AS-MOE fails to operate whereas AS-CMOE performs well and robustness to mismatch is shown.

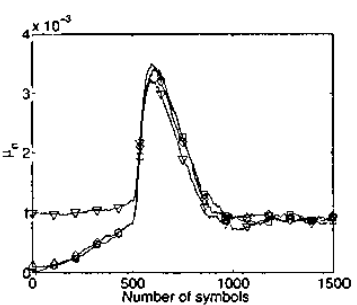

a)

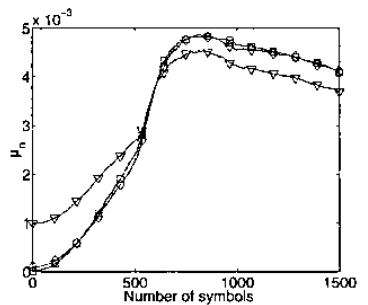

b)
Fig. 2. The trajectories of step-sizes for the AS-CMOE receiver in a) the single-path b) the five-ray multipath channels when initial step-sizes are (i) $1 \times 10^{-5}$ for $-\square-$ (ii) $1 \times 10^{-4}$ for $-\diamond-$ and (iii) $1 \times 10^{-3}$ for $-\nabla-$. The trajectories of the step-size show an approximately identical behaviour. All trajectories of the step-size for the AS-CMOE receiver behave similarly after $n=500$ and stay at the same level after $n=1000$.

transition probability matrix $\mathbf{P}=\left(\begin{array}{ccc}0.998 & 0.01 & 0.01 \\ 0.01 & 0.998 & 0.01 \\ 0.01 & 0.01 & 0.998\end{array}\right)$ and state space $\left\{(1,0,0)^{T},(0,1,0)^{T},(0,0,1)^{T}\right\}$. The Markov chain $x[n]$ designates the MAI user group which affects the desired user detection at the $n$th symbol. The switching between the three MAI user groups occurred at every 500 symbols. Single-path fading channels were used for both the desired user and all the MAI users for all user groups. At different adaptation rate $\alpha$ settings, Fig. 3 shows the different trajectories of the step-size $\mu_{n}$ of AS-CMOE. The initialisation of step-size $\mu_{0}$ was set at $10^{-6}$ and the adaptation rates at $\alpha=\left\{2 \times 10^{-8}, 1 \times 10^{-7}, 5 \times 10^{-7}\right\}$. Each plot was averaged over 50 different runs for $8 \times 10^{4}$ symbols. From the plots, the trajectories of the step-sizes converge to an approximately similar level despite a 25 -fold difference in adaptation rate setting. The step-sizes converge to some small levels but will not go to zero. Although unique global convergence has not yet been proven, the consistency in convergence of different adaptation rates suggests the insensitivity of adaptation rate $\alpha$ of AS-CMOE.

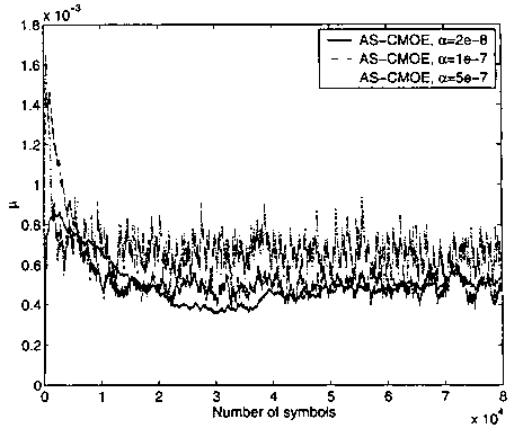

Fig. 3. The trajectories of step-sizes of AS-CMOE in Markovian user population with different settings of adaptation rate $(\alpha)$. The AS-CMOE algorithm is shown to be relatively insensitive to variation of adaptation rate settings.

\section{CONCLUSION}

In this paper, the AS-CMOE receiver is introduced. The performance of the algorithm for nonstationary environments within singlepath and multipath fading channels is examined. The algorithm works well in tracking the abrupt changes due to interfering users entering and exiting the channels. AS-CMOE performs closely to AS-MOE of [4] in the single-path channel. However, as the ASMOE receiver fails to operate in the multipath channel, AS-CMOE performs well in tracking abrupt changes and its robustness to mismatch is shown. The insensitivity to the initialisation points of step-size and adaptation rate of AS-CMOE are also investigated. Simulation results suggest the relative insensitivity to variation of both initial step-size and adaptation rate settings of the AS-CMOE algorithm.

\section{REFERENCES}

[1] M. L. Honig, U. Madhow, and S. Verdú, "Blind adaptive multiuser detection," IEEE Trans. Inform. Theory, vol. 41, no. 4, pp. 944-966, 1995.

[2] M. K. Tsatsanis, "Inverse filtering criteria for CDMA systems," IEEE Trans. Signal Processing, vol. 45, no. 1, pp. 102-112, 1997.

[3] P. Yuvapoositanon, Blind Adaptive Techniques for DirectSequence Code Division Multiple Access Receivers, Ph.D. thesis, Imperial College, University of London, England, 2002.

[4] V. Krishnamurthy, G. Yin, and S. Singh, "Adaptive step-size algorithms for blind interference suppression in DS/CDMA systems," IEEE Trans. Signal Processing, vol. 49, no. 1, pp. 190-201, 2001

[5] H. J. Kushner and J. Yang, "Analysis of adaptive step-size SA algorithms for parameter tracking," IEEE Trans. Automat. Contr., vol. 40, no. 8, pp. 1403-1410, 1995.

[6] V. J. Matthews and Z. Xie, "A stochastic gradient adaptive filter with gradient adaptive step size," IEEE Trans. Signal Processing, vol. 41, no. 6, pp. 2075-2087, 1993. 